(C) [2010] IEEE. Reprinted, with permission, from [Jaime VallsMiro, A kyno-dynamic metric to plan stable paths over uneven terrain, Intelligent Robots and Systems (IROS), 2010 IEEE/RSJ International Conference on, 18-22 Oct. 2010]. This material is posted here with permission of the IEEE. Such permission of the IEEE does not in any way imply IEEE endorsement of any of the University of Technology, Sydney's products or services. Internal or personal use of this material is permitted. However, permission to reprint/republish this material for advertising or promotional purposes or for creating new collective works for resale or redistribution must be obtained from the IEEE by writing to pubs-permissions@ieee.org. By choosing to view this document, you agree to all provisions of the copyright laws protecting it 


\title{
A Kyno-dynamic Metric to Plan Stable Paths Over Uneven Terrain
}

\author{
Jaime Valls Miró*, Gaultier Dumonteil**, Christoph Beck*** and Gamini Dissanayake*
}

\begin{abstract}
A generic methodology to plan increasingly stable paths for mobile platforms travelling over uneven terrain is proposed in this paper. This is accomplished by extending the Fast Marching level-set method of propagating interfaces in 3D lattices with an analytical kyno-dynamic metric which embodies robot stability in the given terrain. This is particularly relevant for reconfigurable platforms which significantly modify their mass distribution through posture adaptation, such as robots equipped with manipulator arms or varying traction arrangements. Results obtained from applying the proposed strategy in a mobile rescue robot operating on simulated and real terrain data illustrate the validity of the proposed strategy.
\end{abstract}

\section{INTRODUCTION AND MOTIVATION}

One of the many application domains where mobile robots are deployed is on environments which are inaccessible or considered too dangerous for humans to operate in, such as those frequently encountered by planetary exploration rovers, mining or search and rescue robots whose navigation does not generally occur over well-known, flat, homogeneous terrains. On the contrary, platforms have to deal with rough, uneven surfaces and many uncertainties, and these characteristics have a strong influence on the robot's ability to perform as planned. For the specific case of reconfigurable robots operating under these conditions, their kinematic configuration also plays a crucial factor in the interaction between vehicle and terrain, and having the ability to actively assume safer poses that reduce potential instabilities, such as those leading to vehicle tip-over, is a desirable feature. Various stability criterions have been proposed in the literature to analyse the qualitative performance of robot stability, mostly with the aim of real-time short-term tip-over monitoring, prediction and prevention, or off-line trajectory optimisation. In this study, a stability measure is also employed to provide a reliable measure for the stability about each tip-over axis of the robot. However, based on this analysis, a novel variational formulation of the classical Fast Marching Method is proposed to enhance the safe traversability of the resulting path over irregular terrain. The proposed algorithm thus combines the remarkable computational properties of the wavefront propagation proposed by the Fast Marching method, with the non-Euclidean metrics derived from the vehicle stability constraints, to extract traversable stable paths for the given three-dimensional environment.

\footnotetext{
*Faculty of Engineering and IT, University of Technology Sydney (UTS), Sydney NSW 2007, Australia javalls, gdissaleng.uts.edu.au

**IUP Intelligent Systems, University Paul Sabatier, Tolouse, France gautier.dumonteilewanadoo.fr

***Faculty of Mechanical Engineering, University of Karlsruhe, Germany chirstoph.beckestudent.kit.edu
}

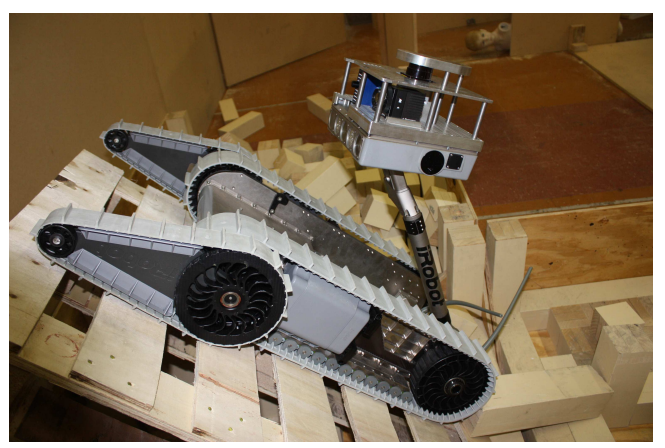

Fig. 1: The iRobot PackBot robot with sensor payload unit, in a mock-up Urban Search And Rescue (USAR) test arena.

In this work, the proposed planning strategy is illustrated with the quasi-static model of the multi-tracked iRobot PackBot platform, mounted with an arm and a pan and tilt sensor-head unit, as depicted in Figure 1.

\section{LEVEL SET METHODS FOR PATH PLANNING}

The Fast Marching Method (FMM) [1] is an efficient numerical method for solving boundary value partial differential equations in the general context of level set methods for propagating interfaces. The FMM gives the evolution of a continuous front wave in an inhomogeneous medium, whose travel-time is governed by an (approximate) solution to a well-understood non-linear continuous mathematical formula known as the Eikonal equation. The minimal length properties of the resulting geodesic paths have a wide range of applications, including problems in fluid mechanics, combustion, computer animation, image processing, or the structure of snowflakes to name a few.

The extraction of shortest paths has been extensively surveyed in the literature, and it is not the objective of this paper. However, some background is hereby provided, which is by no means comprehensive but for the benefit of contextualising the FMM. Probably the canonical method for computing shortest paths on graphs or discretised settings is Dijkstra's algorithm [2]. To speed up the computations, some heuristics have been proposed that reduced the search space, and the $\mathrm{A}^{*}$ algorithm is extensively used [3], particularly when a path from a given start point to a known goal needs to be calculated in real-time. However, it should be noted that $\mathrm{A}^{*}$ requires different searches for each pair of start and goal points. Other tree-search strategies, such as IDA* [4], have also been proposed. For the case of Euclidean metrics, the exploitation of specific data structures have also given rise to faster algorithms, such as visibility graphs [5]. 
Despite saving in processing time, in general terms a notable constraint of these discrete-computation methods is the need of "smoothing" operators to produce realistic paths, as the solutions need to follow existing gridding connections. It is clear that the breadth-first nature of the FMM search has an undeniable weakness in the computing overhead when compared with other methods. In contrast, it also exhibits a number of strengths which make it rather attractive to extract geodesics, particularly for three-dimensional data sets : it can be used with non-Euclidean (and continuous) metrics, it can find geodesic paths that follow arbitrary directions, and it is guaranteed to find the optimal paths to each and every one of the units defining the search space, a particularly precious resource in active exploration.

Alternatives to save processing time by heuristically restricting the FMM front propagation between a given pair of points have also been proposed [6].

\section{A. The Fast Marching Method (FMM)}

The following is a brief description of the FMM. For a detailed description, the reader is referred to [1]. The FMM has been typically applied in problems which deal with evolving fronts, such as seismology. The analogy with seismic theory is rather effective to understand the fundamentals of the FMM [7]. Topographic terrains provide information about elevation of the surface above sea level by contour lines. Each point on a contour line has the same elevation, so a contour line represents an equipotential curve. A set of contour lines tells the trained interpreter the shape of the terrain: hills are represented by concentric loops, stream valleys by vees, steep slopes have closely spaced contour lines, gentle slopes have widely spaced contour lines. The contour interval is the elevation difference between adjacent contour lines. In seismic theory, the travel-time distance surface $T(\mathbf{x})$ is a function of the spatial terrain coordinates that is analogous to the potential, whereas its contour curve represents the propagating wavefronts. The gradient vector is perpendicular to the contour curve, and its magnitude indicates the steepness of the slope. The geodesic curve $\mathbf{r}(t)$ traced out by the seismic energy vector moves in such a manner that its direction at any point coincides with the direction of the gradient at that point. The FMM makes use of the fact that this motion relationship satisfies the non-linear Eikonal equation, which in scalar form is given by:

$$
\|\nabla T(\mathbf{x})\|=s(\mathbf{x})
$$

where the left side implies the wavefront of the potential, and the right side the reciprocal of the seismic velocity, or slowness, a weighting factor dictated by the given terrain.

Hence, given $s(\mathbf{x})$, the weighted geodesic distance between two points $x_{0}, x_{1} \in \mathbb{R}^{d}$ can be defined as

$$
d\left(x_{0}, x_{1}\right)=\min _{r}\left(\int_{0}^{1}\|\mathbf{r}(t)\| s(\mathbf{r}(t)) \mathrm{d} t\right)
$$

where $\mathbf{r}(0)=x_{0}$ and $\mathbf{r}(1)=x_{1}$. It is worth noting that when $s=1$, the integral in (2) corresponds to the length

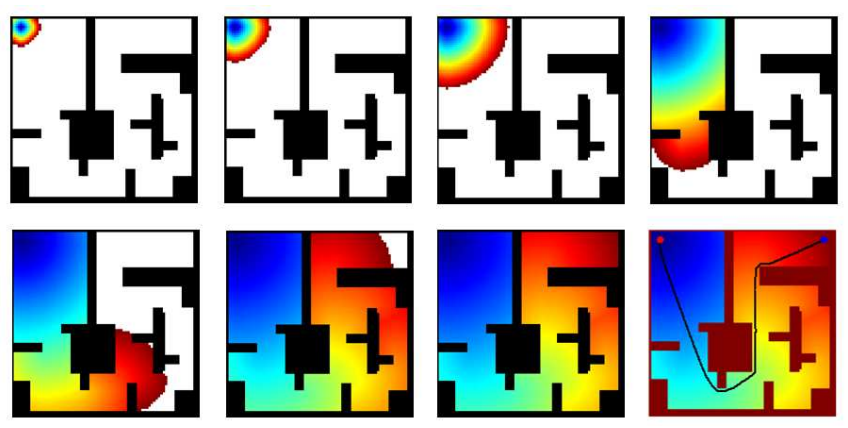

Fig. 2: Shortest FMM path on a 2D scenario, path cost depicted as colour gradient wave from blue to red.

of the curve $\mathbf{r}(t)$ and therefore $d$ is the classical Euclidean distance.

The key feature behind the FMM is a careful selection of the grid points when evaluating the travel time. This order is based on the causality relationship, which states that the arrival time $t$ at any point depends only on the neighbours that have smaller values. During the evolution of the front, each grid point $\mathbf{x}$ is assigned one of the three possible tags:

1) Known: the computed travel time at $\mathbf{x}$ will not be changed later.

2) Narrow-Band (or trial set): the computed travel time at $\mathbf{x}$ may be changed later.

3) Far-Away: the travel time at $\mathbf{x}$ is not yet computed and initially set with infinitely large values.

The procedure to update $T(\mathbf{x})$ for a given point $\mathbf{x}_{i}$ is based on the upwind first-order approximation to solve (1), a quadratic equation given by:

$$
\sum_{j}=\left(\frac{T\left(\mathbf{x}_{i}\right)-T\left(\mathbf{x}_{j}\right)}{\Delta x_{i j}}\right)^{2}=s^{2}\left(\mathbf{x}_{i}\right)
$$

where $j$ is the (variable) number of neighbouring points and $\Delta x_{i j}$ the grid size in the $i j$ direction (depends on the dimensionality of the problem).

In simple terms, all initial points (one in case of the front emanating from a single point, some other arbitrary shape otherwise) are tagged as Known. Then, their nearest neighbours are tagged as Narrow-Band after computing their arrival time by solving (3) and the fact that a grid's point arrival time gets updated by neighbouring points with smaller travel-times only. This monotonicity property allows for the maintenance of a small Narrow-Band of candidate points around the front representing its outward motion. Arrangement of the elements of Narrow-Band in a heap is often used in the implementations to efficiently track multiple propagating fronts emanating from different points in the domain.

As a result of the updating, either a Far-Away point is marked as Narrow-Band, or a Narrow-Band is assigned a new value. When all points have been visited (and unlike other front propagation algorithms each grid point is visited only once [8]), the geodesic curve can then be computed by 
the back-propagation of the steepest gradient descent. Note that this is a local computation, and only uses the value of $T(\mathbf{x})$ for a small fraction of the visited grid points, all located within the Known set at the end of the front propagation procedure. An example of the evolution of the FMM on a $2 \mathrm{D}$ environment with walls and empty rooms is depicted in Figure 2. It can be observed how the resulting path is not necessarily the safest but the shortest one.

\section{STABILITY METRIC}

There have been a number of propositions to address the issue of stability in mobile robots. Some research has focused on the analysis of the robot's Centre of Gravity (CoG) to find suitable controls to cope with specific scenarios like overcoming obstacles and small ditches [9] or climbing stairs [10]. A multi tracked robot on a steep slope was examined in [11] to determine boundaries for the CoG and came up with a strategy to traverse a given slope. A stability margin measure was introduced by Papadopoulos and Rey [12] to estimate the predicted time until tip-over for large mobile manipulator robots, such as forestry vehicles. They also recommended stabilising steps by using certain actuators. Stability indices have understandably played a decisive role in the history of walking robots, and a number of measures have been proposed in the literature (e.g., the Static Stability Margin [13] or the Energy Stability Margin [14]). More general approaches for the stability control of reconfigurable mobile robots have also taken into account other constraints such as traction optimisation [15], [16]. In both works, the original Force-Angle Stability Margin (FASM) [12] was used. While that proved sufficient for platforms with relatively high and not significantly changing CoG's, it was nevertheless not a representative measure of stability for many other robot configurations, such as those more strongly subjected to external forces and moments. A revised version to the original FASM was subsequently proposed by Papadopoulos and Rey to allow for dynamical changes in the robot configuration [17]. It also constitutes a more suitable stability measure for mobile robots/manipulators as it exhibits a more simplistic geometric interpretation and thus could be more easily computed. It is for this reason that this is the metric employed in this work. As the metric was introduced in two different versions, these are briefly reviewed in the next Section to better understand the influence of the CoG's height for platforms that can significantly reposition their centre of mass to improve stability in uneven terrains.

\section{A. The Force-Angle Stability Margin (FASM)}

The FASM measure $\beta$ was first proposed in 1996 [12] as

$$
\beta=\min \left(\theta_{i}\left\|\mathbf{f}_{\mathbf{i}}\right\|\right)
$$

where $\mathbf{f}_{\mathbf{i}}$ is the net force (including all static and dynamic forces, as well as moments) contributing to a potential rollover about a particular tip-over axis $\mathbf{a}_{\mathbf{i}}$. The tip-over axes $\mathbf{a}_{\mathbf{i}}$ are given as the lines between $m$ arbitrary supporting points $\mathbf{p}_{\mathbf{i}}, i=\{1, . ., m\}$

$$
\mathbf{a}_{\mathbf{i}}=\mathbf{p}_{\mathbf{i}+\mathbf{1}}-\mathbf{p}_{\mathbf{i}}, i=\{1, \ldots, m-1\}
$$

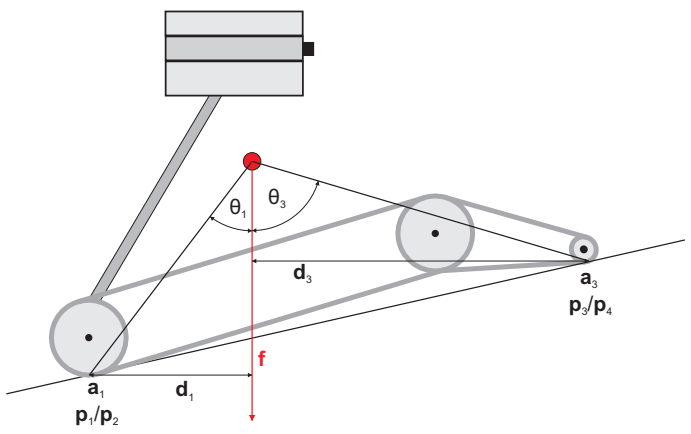

Fig. 3: Example FASM in 2D.

$$
\mathbf{a}_{\mathbf{m}}=\mathbf{p}_{1}-\mathbf{p}_{\mathbf{m}}
$$

$\theta_{i}$ is the angle between $\mathbf{f}_{\mathbf{i}}$ and the tip-over axis normal through the tip-over axis and the $\mathrm{CoG}$. Figure 3 illustrates these parameters in a two dimensional example, where $\mathbf{a}_{\mathbf{1}}$ and $\mathbf{a}_{3}$ are perpendicular to the paper representing the tipover axes through $\mathbf{p}_{\mathbf{1}} / \mathbf{p}_{\mathbf{2}}$ and $\mathbf{p}_{\mathbf{3}} / \mathbf{p}_{\mathbf{4}}$ respectively.

The revised version of FASM was published in 2000 [17] and besides $\mathbf{f}_{\mathbf{i}}$ and $\theta_{i}$ also included $\mathbf{d}_{\mathbf{i}}$, the distance between $\mathbf{a}_{\mathbf{i}}$ and $\mathbf{f}_{\mathbf{i}}$ as

$$
\beta=\min \left(\theta_{i}\left\|\mathbf{d}_{\mathbf{i}}\right\|\left\|\mathbf{f}_{\mathbf{i}}\right\|\right)
$$

This enables the metric to become sensitive to varying heights of the CoG. The greater the value of the stability measure $\beta_{i}$, the more stable the vehicle becomes in terms of tipping over about the given axis. Negative values of the measure indicate an occurring tip-over instability.

The tip-over axis normal $\mathbf{l}_{\mathbf{i}}$ that intersects the CoG is given by

$$
\mathbf{l}_{\mathbf{i}}=\left(I-\hat{\mathbf{a}}_{\mathbf{i}} \hat{\mathbf{a}}_{\mathbf{i}}^{\mathbf{T}}\right)\left(\mathbf{p}_{\mathbf{i}+\mathbf{1}}-\mathbf{p}_{\mathbf{C o G}}\right)
$$

where $\hat{\mathbf{a}}_{\mathbf{i}}$ is the normalised vector of $\mathbf{a}_{\mathbf{i}}, \mathbf{p}_{\mathbf{C o G}}$ is the position of the $\mathrm{CoG}$ and $I$ is the $3 \times 3$ identity matrix.

Given $f_{r}$, the net force acting on the CoG which includes gravitational, external and inertial forces, and $\mathbf{n}_{\mathbf{r}}$, the net moment encompassing all external and inertial moments about the CoG axis, the effective net force $\mathbf{f}_{\mathbf{i}}$ that contributes to a potential tip-over about one specific axis $\mathbf{a}_{\mathbf{i}}$ can be determined by

$$
\mathbf{f}_{\mathbf{i}}=\left(I-\hat{\mathbf{a}}_{\mathbf{i}} \hat{\mathbf{a}}_{\mathbf{i}}^{\mathbf{T}}\right) \mathbf{f}_{\mathbf{r}}+\frac{\hat{\mathbf{l}}_{\mathbf{i}} \times\left(\left(\hat{\mathbf{a}}_{\mathbf{i}} \hat{\mathbf{a}}_{\mathbf{i}}^{\mathbf{T}}\right) \mathbf{n}_{\mathbf{r}}\right)}{\left\|\mathbf{l}_{\mathbf{i}}\right\|}
$$

The first term considers the part of the net force perpendicular to the tip-over axis. The second term considers the moment that participates about the tip-over axis, converted into an equivalent force couple, where one member of the couple passes through the CoG and thus can be added to the net force, whereas the other member passes through the tip-over axis and hence does not contribute to $\mathbf{f}_{\mathbf{i}}$. by

The angle $\theta_{i}$ for each tip-over axis can then be computed

$$
\theta_{i}=\sigma_{i} \cos ^{-1}\left(\hat{\mathbf{f}}_{\mathbf{i}} \hat{\mathbf{l}}_{\mathbf{i}}\right)
$$




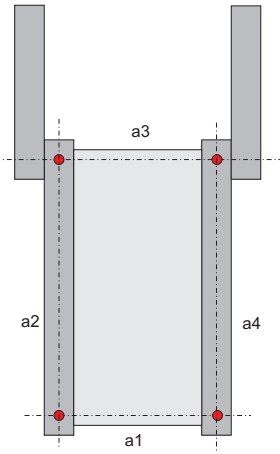

(a) Flippers up contact points.
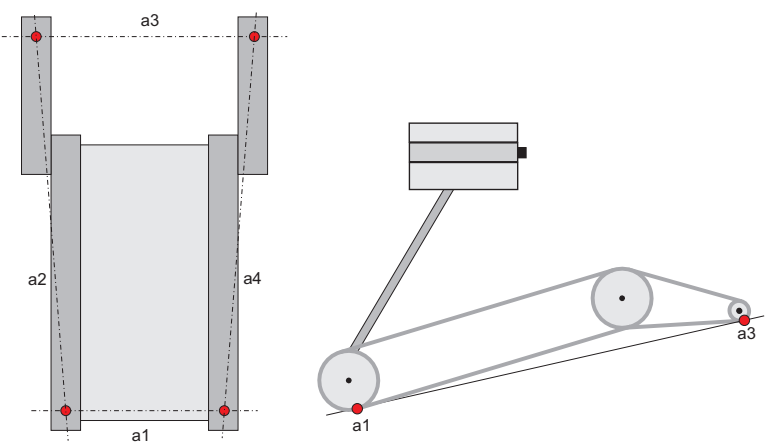

(b) Flippers down contact points.

Fig. 4: Surfaces defined by the contact points used to calculate the robot Centre of Gravity.

where

$$
\sigma_{i}= \begin{cases}+1 & \left(\hat{\mathbf{f}}_{\mathbf{i}} \times \hat{\mathbf{l}}_{\mathbf{i}}\right) \hat{\mathbf{a}}_{\mathbf{i}}>0 \\ -1 & \text { otherwise }\end{cases}
$$

The revised FASM also requires the shortest distance $\mathbf{d}_{\mathbf{i}}$ between $\mathbf{a}_{\mathbf{i}}$ and $\mathbf{f}_{\mathbf{i}}$, which can be obtained by adding the projection of $\mathbf{l}_{\mathbf{i}}$ on $\mathbf{f}_{\mathbf{i}}$ to negative $\mathbf{l}_{\mathbf{i}}$, i.e.

$$
\mathbf{d}_{\mathbf{i}}=-\mathbf{l}_{\mathbf{i}}+\left(\mathbf{l}_{\mathbf{i}}^{\mathbf{T}} \hat{\mathbf{f}}_{\mathbf{i}}\right) \hat{\mathbf{f}}_{\mathbf{i}}
$$

For more details on these derivations, the reader is referred to [17].

\section{B. Normalisation}

The stability measure for a given configuration is normalised over the weakest tip-over axis in the most stable "home" robot pose (flat on the horizontal floor, with arm and flippers folded for the PackBot). Normalisation facilitates the general interpretation of the stability measure independently of the vehicle type, and permits meaningful comparisons based on the stability measures, for different vehicles or for different terrains, an important factor in this work.

\section{Robot Model}

The PackBot robot depicted in Figure 1 was the platform employed to validate the practical aspects of this research. It consists of a skid-steer vehicle base, equipped with two front flippers that enable the robot to traverse obstacles and rough terrain. A manipulator arm attaches to the vehicle base via a 1 Degree of Freedom (DoF) shoulder joint. It carries a 2-DoF pan-and-tilt unit equipped with several cameras and lights. An additional sensor head unit is also mounted on top of the arm head to enhance the search and rescue capabilities of the robot in its navigational and victim identification activities. It incorporates a laser scanner, a 3D time-of-flight camera and a thermal camera. The robot is battery powered and features two battery compartments on its left and right hand side. Communication with the operator control unit is wireless.

A modelling of the robot serves as the foundation for the application of the FASM. As expressed in (9), this is captured via the computation of the $\mathrm{CoG}$ based on the robot posture and the consideration of the dynamic effects that arise during robot motion. The CoG obtained in the robot frame is given by:

$$
{ }^{R} C o G=\frac{\sum_{i=1}^{n} p_{m_{i}} m_{i}}{m_{\text {tot }}}
$$

where $p_{m_{i}}$ is the position of the lumped mass $m_{i}$ and $m_{t o t}$ is the total robot mass.

The influence of head panning and tilting on the robot's CoG is very small in comparison to the effects that arise from the position of the arm and flippers, and have therefore been neglected here. Thus, the arm and the flipper poses are the key reconfigurable DoFs considered. While the formulation allows for full dynamic effects to be readily incorporated, as the rover is operated at very low speeds in Search and Rescue operations only results with static forces are presented in this work.

The contact footprint between the platform and the surface is defined by four points. They are assumed to be lengthwise symmetrical, depicting two possible convex quadrilateral contact surfaces as shown in Figure 4. Two contact points are always fixed at the robot base rear sprocket. As flippers operate simultaneously on the PackBot, the other two contacts are chosen based on the flipper pose. When the flippers touch the ground, the front contact is at the flipper's front sprockets, defining an isosceles trapezoid as depicted in Figure $4 \mathrm{~b}$. When the flippers do not interact with the terrain, the front contact is assumed to be at the robot base front sprockets, thus describing a rectangular area, as shown in Figure $4 \mathrm{a}$. The connecting lines between the ground contacts represent the tip-over axes $a_{i}, i=\{1, . ., 4\}$.

\section{The Stable FMM Algorithm for Planning in 3D LATTICES}

Incorporating the computations for the stability of the robot into the FMM path planning framework constitutes the main proposition of this paper. By encouraging the robot to move along paths of higher stability instead of arbitrary metrics, as those illustrated in Figure 5, the planning stage is shifted towards finding paths that quantitatively aim to guarantee the stability of the robot for a given terrain.

This has been accomplished by merging the stability margins into the FMM potentials through the environmental 


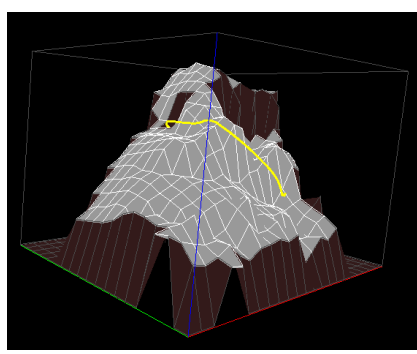

(a) FFM shortest path.

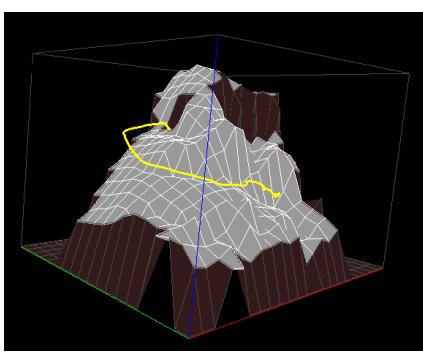

(b) FFM with vertical penalty.
Fig. 5: FMM paths (in yellow) on the same 3D real scenario with two potentials: shortest topographical path on the left, and with an arbitrary vertical displacement penalty.

slowness factor $s(\mathbf{x})$ in (1). The steps are as follows: the set of data points that represent the terrain to be traversed by the robot is first tessellated using Delaunay triangulation (see, for example, Figure 6a). Among other possible triangulation alternatives, Delaunay is preferred for mesh generation as it maximises the minimum triangulation angles. In practice, this means that no data points are contained in the circles circumscribing each triangle. It is assumed in this work that each planar tessellate is sufficiently large to span the robot contact points with the terrain. Having obtained the slope for each surface in the mesh, the elevation and bank angles of the robot at an arbitrary number of possible orientations within can be calculated for the entire environment, and fed as required to the FASM. Four orientations have been computed in the results presented here, although finer orientations are equally attainable. Since anticipated orientations can not be computed until the path is generated and the vehicle is unconstrained to traverse the mesh surface in any orientation, the number of orientations is an arbitrary decision to coarsely cover the whole spectrum of vehicle orientations. Many feasible alternatives are possible, such as for instance a variable discretization number based on how levelled each mesh surface is. While some have been tested, they do not add to the discussion and general results, are have not been included for brevity.

An example of the resulting environment potential for the artificial terrain shown in Figure 6a is depicted in Figure 6c, where colour-coding has been used to visualize in each given 2D lattice the best of the stability margins for the four robot orientations considered here, or black if the robot is found to be unstable. Blue equals North, yellow represents South, green is East and red represents West. This terrain representation is then employed by the proposed FMM to propagate the wavefront and find optimally stable paths.

\section{A. Results and Analysis}

Two distinctive examples are provided to show the proposed algorithm in operation in challenging and representative environments. Results depicted in Figure 6 have been artificially created to better illustrate the advantages of the proposed metric. A ramp has been added to a walled en-

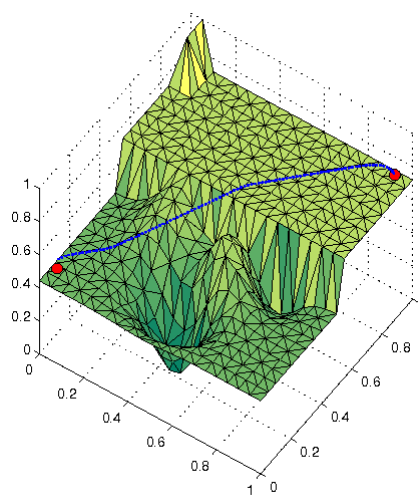

(a) Shortest geodesic.

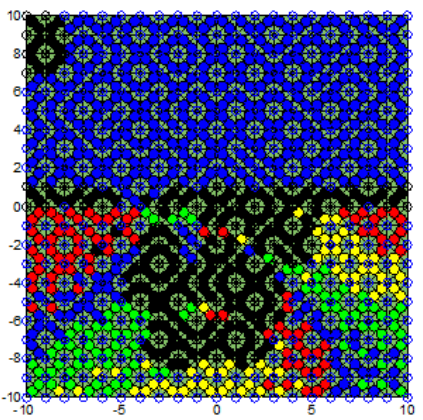

(c) Stability map.

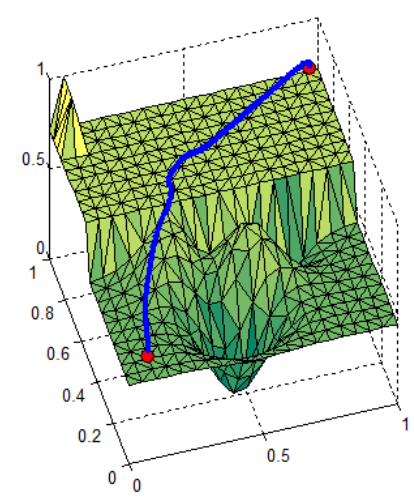

(b) Most stable geodesic 3D.

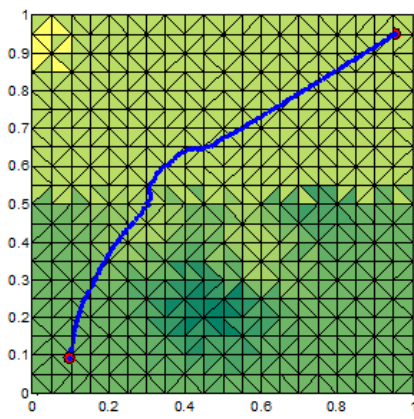

(d) Most stable geodesic 2D view.
Fig. 6: Example of stable geodesic paths on simulated data.

vironment. It can be seen in Figure 6a how the standard wavefront propagation would miss the preferred route, as it is not the shortest path, jeopardising the balance of the platform over the wall. A specific potential for this terrain could also be found that would follow the exit ramp, for instance penalising large discontinuities. However, this is not necessarily a measure applicable to generic environments. On the other hand, the proposed methodology is able to exploit robot reconfigurations along the geodesic path in a generalised way to confidently derive stable paths, as depicted in Figure $6 b$.

The algorithm has also been applied to point cloud data obtained from the range camera mounted on the Packbot, deployed in a traditional USAR scenario as the one depicted in Figure 1. Results representative of those obtained are collected in Figure 7, where it can be seen how the proposed planner is able to come up with more stable (if generally more winding) geodesics than those where no stability is accounted for. Cost and grid length for each of the token paths considered are collected in Table I. Potentials have been normalized with respect to largest value, so that in both instances a value of 1 is largest (in the case of the standard FMM this is the equi-potential value used to plan throughout the entire terrain). While this allows for a more meaningful comparison of the terms, the real measure of comparison is the inability of the shortest geodesic to travel over a terrain that guarantees a stable pose for the platform 


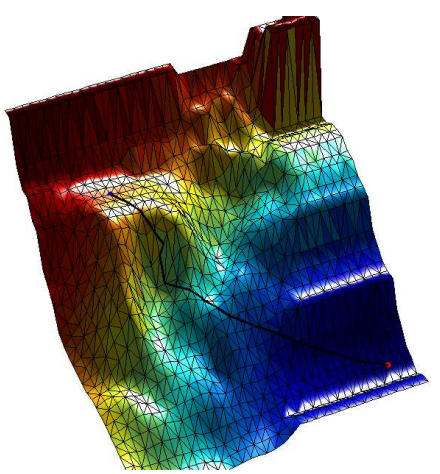

(a) Shortest geodesic.

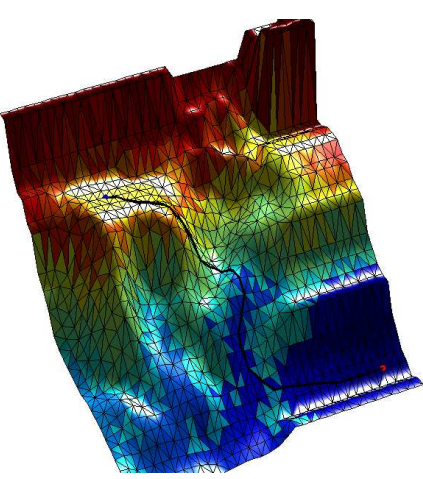

(b) Most stable geodesic.
Fig. 7: Shortest and most stable geodesics on real terrain data. Colour coding cost increases from blue to red.

TABLE I: Comparison of FMM paths

\begin{tabular}{|c||c|c|c|}
\hline Potential & Grid path length & Cost & Guaranteed stability \\
\hline Constant & 32 & 16.66 & No \\
\hline Stability & 35 & 13.14 & Yes \\
\hline
\end{tabular}

along the path. On the contrary, the shortest path in the given example would result in the platform tipping over, no matter what configuration is assumed. Inducing stability in the planner via the kyno-dynamic metric hereby proposed guarantees that, if a path exists, the resulting geodesic will be stable.

\section{B. Shortcomings}

The proposed planning mechanism constitutes a formalisation of a stability metric in order to supplement geometric planning. Being intrinsically based on a weighting factor, it is important to raise awareness about the sensitivity of the solution to variations in gridding or the choice of stability scaling, both of which would results in different paths. While results indicate that suitable stable geodesics can be practically computed for a given gridding pattern, this is nevertheless an issue that is being investigated further, as there is no known generalised solution to overcome this limitation at this stage.

\section{CONCLUSIONS AND FUTURE WORKS}

A path planner aimed at improving the safe deployment of mobile platforms when operating in uneven terrain has been proposed in this work. The methodology is particular applicable, although not restricted to, reconfigurable platforms that can actively engage in safer poses to reduce potential instabilities, such as those leading to vehicle tipover. A variational computation based on the Force-Angle Stability Measure has been proposed in combination with the Fast Marching Level Set Method to reliably generalise robot instabilities and derive more balanced paths for the robot to follow. Simulations with real and artificial 3D data sets have been provided to demonstrate the performance of the algorithm for a tracked vehicle operating in rough terrain.
Future work includes accounting for full dynamics and the addition of other parameters known to jeopardise reliable robot operation, such as slippage. Both the assumption of a symmetrical footprint, and one that fits in the meshed triangles are known to be oversimplifications when operating in highly unstructured terrain, and work is currently underway to soften this restrictions. Inferring data when working with incompletely sensed terrains and the use of "constrained" triangulation to preserve features such as edges are also highly desirable to yield feasible and stable paths.

\section{ACKNOWLEDGMENTS}

This work is supported by the Australian Research Council through its Centre of Excellence programme, and by the New South Wales State Government.

\section{REFERENCES}

[1] J. A. Sethian, Level Set Methods and Fast Marching Methods Evolving Interfaces in Computational Geometry, Fluid Mechanics, Computer Vision, and Materials Science. Cambridge University Press, 1999.

[2] E. W. Dijkstra, "A note on two problems in connexion with graphs," Numerische Mathematik, vol. 1, pp. 269-271, 1959.

[3] N. Nielsson, Problem-Solving Methods in Artificial Intelligence. McGraw-Hill, 1971.

[4] R. Korf, "Depth-first iterative-deepening: An optimal admissible tree search," Artificial Intelligence, vol. 27, no. 1, pp. 97-119, 1985.

[5] H. Rohnert, "Shortest path in the plane with convex polygonal obstacles," Information Processing Letters, vol. 23, no. 2, pp. 71-76, 1986.

[6] G. Peyre and L. Cohen, "Heuristically driven front propagation for fast geodesic extraction," Computational Vision and Biomechanics, vol. 1, no. 1, pp. 55-67, 2008.

[7] E. Robinson and D. Clark, "Basic seismology 14 - michael faraday and the eikonal equation," The Leading Edge, Society of Exploration Geophysicists, vol. 26, no. 1, pp. 24-26, 2007.

[8] O. Cuisenaire, "Distance transformations: Fast algorithms and applications to medical image processing," Ph.D. dissertation, Univ. Catholique de Louvain, Belgium, 1999.

[9] W. Wang, Z. Du, and L. Sun, "Dynamic load effect on tracked robot obstacle performance," in Proc. of International Conference on Mechatronics WA1-B-4, Kumamoto, Japan, 2007.

[10] P. Ben-Tzvi, S. Ito, and A. A. Goldenberg, "Autonomous stair climbing with reconfigurable tracked mobile robot," in Proc. IEEE International Workshop on Robotic and Sensors Environments, Ottawa, Canada, 1213 October 2007, 2007.

[11] S. Shoval, "Stability of a multi tracked robot traveling over steep slopes," Proc. IEEE International Conference on Robotics and Automation, New Orleans, LA, USA, 2004.

[12] D. A. Rey and E. G. Papadoupoulos, "A new measure of tipover stability margin for mobile manipulators," Proc. IEEE International Conference on Robotics and Automation, Minneapolis, Minnesota, April, 1996.

[13] R. McGhee and A. Frank, "On the stability properties of quadruped creeping gaits," Mathematical Bioscience 3, vol. 3, pp. 331-351, 1968.

[14] D. A. Messuri and C. Klein, "Automatic body regulation for maintaining stability of a legged vehicle during rough-terrain locomotion," IEEE Trans. Robot. Automat., vol. 3, pp. 132-141, 1985.

[15] P. Schenker, T. Huntsberger, P. Pirjanian, S. Dubowsky, K. Iagnemma, and V. Sujan, "Rovers for agile, intelligent traverse of challenging terrain," Proceedings of the 7th International Symposium on Artificial Intelligence, Robotics and Automation in Space, i-SAIRAS, Nara, Japan, May, 2003.

[16] G. Besseron, C. Grand, F. B. Amar, and P. Bidaud, "Decoupled control of the high mobility robot hylos based on a dynamic stability margin," in Proc. IEEE/RSJ International Conference on Intelligent Robots and Systems, Nice, France, September 22-23, 2008.

[17] D. A. Rey and E. G. Papadoupoulos, "The force angle measure of tipover stability margin for mobile manipulatiors," Vehicle System Dynamics, vol. 33, pp. 29-48, 2000. 